\title{
Memory and Mystery: The Cultural Selection of Minimally Counterintuitive Narratives
}

\author{
Ara Norenzayan ${ }^{\mathrm{a}}$, Scott Atran ${ }^{\mathrm{b}}$, Jason Faulkner ${ }^{\mathrm{a}}$, Mark Schaller ${ }^{\mathrm{a}}$ \\ a Department of Psychology, University of British Columbia \\ bInstitut Jean Nicod, CNRS, Paris and \\ Institute for Social Research, The University of Michigan, Ann Arbor
}

Received 12 January 2005; received in revised form 19 October 2005; accepted 17 January 2006

\begin{abstract}
We hypothesize that cultural narratives such as myths and folktales are more likely to achieve cultural stability if they correspond to a minimally counterintuitive (MCI) cognitive template that includes mostly intuitive concepts combined with a minority of counterintuitive ones. Two studies tested this hypothesis, examining whether this template produces a memory advantage, and whether this memory advantage explains the cultural success of folktales. In a controlled laboratory setting, Study 1 found that an MCI template produces a memory advantage after a 1-week delay, relative to entirely intuitive or maximally counterintuitive cognitive templates. Using archival methods, Study 2 examined the cognitive structure of Grimm Brothers folktales. Compared to culturally unsuccessful folktales, those that were demonstrably successful were especially likely to fit an MCI template. These findings highlight the role of human memory processes in cultural evolution.
\end{abstract}

Keywords: Culture; Memory; Evolution; Religion; Concepts

\section{Introduction}

What makes a narrative culturally successful? Within any culture, religious tales, folktales, and other narratives are generated by the thousands; but only a few of these tales actually achieve sustained popularity. (We all know "Cinderella," for instance; but "The Donkey Lettuce" never quite caught on.) Of the many ecological and psychological factors that influence the extent to which any such narrative achieves cultural success, mnemonic resilience may be one of the most important. Memorability places necessary constraints on the cultural transmission of narratives and ideas. In oral traditions that characterize most of human cultures throughout history, a narrative cannot be transmitted and achieve cultural success unless it

Correspondence should be addressed to Ara Norenzayan, Department of Psychology, University of British Columbia, 2136 West Mall, Vancouver, BC, Canada V6T 1Z4. E-mail: ara@psych.ubc.ca 
stands the test of memory (Rubin, 1995; Sperber, 1996). Therefore, all else being equal, a more memorable narrative has a competitive advantage over a less memorable one. Because any cultural narrative is likely to go through several generations of repeated transmission and recall, this advantage, even if small at the start, accumulates from generation to generation, leading to massive differences in eventual cultural success.

The psychology of cognition in general, and memory in particular, is thus of great relevance to the anthropological study of how cultural belief systems emerge. Ideas and narratives are not acquired and transmitted through a process by which culture "impinges" on a passive human mind. Rather, the minds of recipients of cultural materials selectively represent, retain, transform, and transmit information. Thus the ordinary biases and transformations in human memory can constrain the content of cultural beliefs. Building on prior research on the cognitive processes that influence the cultural transmission of concepts (e.g., Barrett, 2000; Boyer \& Ramble, 2001), we examine the role of cognitive processes in the cultural transmission of lists of ideas and narratives. We hypothesize that narratives combining mostly intuitive concepts with a minority of counterintuitive ones enjoy a memory advantage, and as a result achieve cultural success. Such an MCI template (Barrett, 2000) may be no accident. Indeed, we propose that it may be a recipe for cultural success: Compared to narratives that fit other templates (e.g., no counterintuitive concepts at all; many counterintuitive concepts), those that are MCI may be especially memorable and, therefore, more likely to achieve cultural stability as well.

\subsection{Intuitive and counterintuitive concepts in cultural narratives}

Before examining the cognitive factors that contribute to the cultural success of narratives, we consider current theorizing and research on the cultural success of various intuitive and counterintuitive concepts that proliferate in cultural narratives. What makes a concept "intuitive" or "counterintuitive"? As several psychologists and anthropologists have noted, the key is whether the concept is consistent with, or violates, ontological assumptions about the properties of ordinary objects (Barrett, 2000; Boyer, 1992; Keil, 1989; see also Atran \& Sperber, 1991).

Intuitive concepts are intuitive because built into them are implicit inferences about their properties. These intuitive inferences are rarely articulated explicitly. Rather, they are assumed, and make the concepts comprehensible and communicable. For example, the concept bird involves the implicit inference that birds fly, that they grow and die, that they drink when thirsty. These inferences are guided by intuitive ontology (Keil, 1989), or core assumptions about the basic categories of existence, such as intentional beings, animals, inanimate objects, and events. Ontology is psychologically important, because it determines the appropriateness of inferences. For example, knowing that birds belong to the ontological category ANIMAL affords "automatic" inferences about biological properties, but not necessarily intentional agent properties. These inferences are possible because ontology is in turn governed by domain-specific "theories" - of mind, biology, and physics - that provide commonsense expectancies and explanations for the workings of each ontological category.

There are important cultural variations in many aspects of domain-specific theories: theory of mind (e.g., Lillard, 1998; Wellman, Cross, \& Watson, 2001), biology (e.g., Medin \& Atran, 1999), and physics (e.g., Peng \& Nisbett, 1998). However, certain core elements of these theo- 
ries appear so early, and are so widespread across human societies, that they may turn out to be psychological primitives that make cultural learning possible. For example, babies as young as 4 months already possess a "theory of physics," having a notion of what counts as a solid object, and assuming, for example, that an object cannot be in different places at the same time, or that a solid object cannot pass through another solid object (Baillargeon, 1998; Carey \& Spelke, 1994; Leslie, 1982; Spelke, 1990). Similarly, preschoolers and adults in most cultures known to anthropologists have a "theory of biology," which dictates that species have biological "essences" and that superficial transformations performed on an animal do not alter its species-specific essence (Atran, 1998; Berlin, 1992; Berlin, Breedlove, \& Raven, 1973; Gelman \& Hirschfeld, 1998; Keil, 1994). Preschoolers and adults across cultures also have an elaborate "theory of mind," which entails, among other things, the attribution of beliefs and desires to people, and the appreciation that people may have false beliefs (Avis \& Harris, 1991; Callahan et al., 2005; Flavell, Zhang, Zou, Dong, \& Qui, 1983; Gardner, Harris, Ohmoto, \& Hamazaki, 1988).

Unlike everyday natural concepts with properties consistent with ontological expectations, there are many other concepts that violate those expectations. Ghosts that walk through walls, frogs that talk, mountains that are invisible to the human eye-these and many other fanciful concepts are inconsistent with intuitive theories of mind, biology, and physics and thus are "counterintuitive." (For broad discussions of intuitive and counterintuitive concepts in culture and religion, see Atran \& Norenzayan, 2004; Barrett, 2000; Boyer, 1994a, 1994b, 2003; Sperber, 1996). Interestingly, despite their obvious incompatibility with ontological expectations-or assumptions about what is actually possible in the real world-these kinds of counterintuitive concepts appear regularly in religious traditions, folktales, myths, and legends around the world (e.g., see Boyer, 1994b, 2001).

\subsection{Past research on the cognitive optimality of MCI concepts}

The effect of unusual or surprising story elements on recall has been examined under the rubric of schema theory, which proposes that recall is a joint product of the interaction between general knowledge structures or expectancies and input information. There is a consensus that schema-relevant information is better remembered than schema-irrelevant information (for reviews, see Alba \& Hasher, 1983; Koriat, Goldsmith, \& Pansky, 2000). However a complex pattern of findings has emerged as to whether recall is enhanced by schema-consistent versus schema-inconsistent information. Incongruous or surprising elements have often been found to produce superior recall under some conditions (e.g., Bower, Black, \& Turner, 1979; Brewer, 1985; Davidson, 1994; McCabe \& Peterson, 1990; Stangor \& McMillan, 1992). Similar effects have been observed in list-learning paradigms, in which conceptually incongruous items enjoy superior recall (e.g., Waddill \& McDaniel, 1998).

However unusual story elements differ from supernatural elements in two important ways. First, unusual story elements disrupt story structure, whereas supernatural elements in a folktale or religion are integral to the story structure (Boyer \& Ramble, 2001). Second, whereas unusual elements in a story involve a schema or script violation that is otherwise consistent with intuitive theories, counterintuitive elements found in folktales, myths, and religions involve ontological violations that are incongruent with intuitive theories. These differences may im- 
plicate different processes and may result in different recall effects (see, for example, Boyer \& Ramble, 2001). Although instructive, these earlier studies on the effect of schema violations have not examined counterintuitive supernatural elements that are recurrent and widespread features of cultures, which is the focus of this research.

One of the earliest accounts of the memorability and transmission of counterintuitive supernatural cultural narratives was Bartlett's (1932) classic study of "the war of the ghosts." Bartlett examined the ways by which British university students remembered and then transmitted a culturally unfamiliar story, in this case a Native American folktale. Interestingly, over several generations of retelling the story, some culturally unfamiliar items or events were dropped from the retelling. Other unfamiliar items were distorted, being replaced by more familiar items. But Bartlett's striking finding was that the very notion of the ghosts-so central to the original story - was gradually eliminated from the retellings, suggesting that counterintuitive elements are at a cognitive disadvantage.

In recent years, there has been growing empirical work on the cognitive factors that constrain the cultural success of beliefs, and this research yields a more complex perspective (Barrett \& Keil, 1996; Barrett \& Nyhof, 2001; Boyer, \& Ramble, 2001). Spirits and other supernatural concepts found in culturally successful narratives (such as religious mythologies) are MCI, having properties that are partially, but not entirely, counterintuitive (Barrett, 2000; Boyer, 2003). Spirits may be invisible or may pass through solid objects; but otherwise they possess the intuitive properties of ordinary intentional agents. Supernatural agents may have supernatural abilities of perception, but they also obey many of the mundane laws of folk physics and folk biology (e.g., they cannot occupy more than one physical location at a time; they get hungry). Indeed, it appears that people assume a substantial set of intuitive properties even for beings that are putatively supernatural: Controlled experiments by Barrett and Keil (1996) indicated that people spontaneously anthropomorphize God in their reasoning, even if doing so contradicts their stated theological beliefs. Culturally successful materials also favor minimal rather than large violations of ontological expectations. In a content analysis of Ovid's Metamorphoses, Kelly \& Keil (1985) found that the ontological transformations experienced by the characters followed a distinct pattern: The number of transformations of one ontological category to other ontological categories decreased as the distance between the two categories increased. Thus, it was far more likely for a conscious being to be transformed into an animal, than a conscious being to be transformed into an inanimate object. Transformations that occur across wide swaths of ontological distance may be just too counterintuitive to be psychologically appealing.

If indeed MCI concepts are cognitively optimal, they should enjoy a cognitive advantage in memory and transmission advantage in communication. Recent studies have supported this conclusion. In a series of experiments, Barrett and Nyhof (2001) asked participants to remember and retell stories containing intuitive, intuitive but bizarre, and counterintuitive events or objects. After 3 generations of retelling the story, the proportion of items recalled in each category was measured. Results indicated that both counterintuitive and intuitive but bizarre items were remembered in greater proportions than intuitive items. Furthermore, the same recall advantage of MCI items was found after a 3-month delay; this is an important finding, given that in most natural settings in which cultural narratives evolve, recall after a long delay is the critical factor. An idea that is memorable immediately, but fades over time, could not be culturally successful. 
Another important finding is that the effect of counterintuitiveness on recall is not linear. Too many ontological violations render a concept too counterintuitive to be comprehensible and memorable. Boyer and Ramble (2001) found that concepts with too many violations were recalled less well than those that were MCI. These results are not only observed immediately after exposure, but also after a 3-month delay - and in cultural samples as diverse as the midwestern United States, France, Gabon, and Nepal (Boyer \& Ramble, 2001). Consistent with the idea that this memory advantage is related to cultural success, the anthropological literature confirms that religious concepts with too many ontological violations are rather rare (Boyer, 1994b). Contrary to the apparent conclusion from Bartlett's classic experiments, these more recent empirical results confirm the idea that MCI concepts are better recalled and transmitted than intuitive ones.

\subsection{Cognitive optimality at the level of narratives}

This existing body of evidence focuses on the extent to which specific narrative elements (e.g., supernatural agents or events) are counterintuitive and the memorability and transmission advantage of those elements. The results imply that MCI elements are especially likely to proliferate in cultural narratives. This implication, however, is inconsistent with the apparent structure of culturally important narratives. If MCI narrative elements enjoy better long-term recall than other concepts, they should dominate religions, folktales, and myths at a much greater rate than is actually observed. However even a casual perusal of culturally successful materials reveals that counterintuitive narrative elements are in the minority. Although counterintuitive concepts regularly appear in popular cultural narratives, the sheer number of them is typically dwarfed by the number of more mundane, intuitive concepts. The Bible, for example, is a succession of mundane events interspersed with a few counterintuitive occurrences, such as miracles and the appearance of angels. In the Grimm Brothers folktales, the tale of "Little Red Riding Hood"- - one of the most celebrated folktales in Western culture-is mostly a series of mundane occurrences, seasoned with only two counterintuitive ones (the talking wolf, and Grandmother and the little girl coming out of the wolf's belly alive). Similarly, the "Beauty and the Beast" has only three such violations (the Beast as an animal with human properties, the magic mirror, and the transformation from beast to human). Why do MCI concepts not dominate the narrative structure of religions, folktales, and myths?

The answer to this apparent puzzle may lie in examining the memorability of a narrative as a single unit of transmission, rather than the individual ideas that are embedded in these narratives. The unit of cultural transmission is often, but not always, an individual concept. Under many conditions, a series of events or ideas are transmitted together as a single unit of culture (Rubin, 1995). Furthermore, recall of individual items is influenced by the narrative context in which the items are embedded (Thorndyke, 1977). Therefore, cognitive optimality might be at work not only at the level of individual narrative elements, but at the level of whole narrative structures as well.

Just as specific narrative elements are especially memorable if they are MCI (Barrett, 2000, Boyer, 2003), so too, whole narratives are likely to be especially memorable if they are MCIif they contain a small number of MCI concepts. If indeed MCI narratives enjoy a memory and transmission advantage (relative to narratives with no counterintuitive elements, or those that 
have an abundance of counterintuitive elements), then cognitive selection would prevent MCI ideas from taking over entire narratives.

Previous research has not addressed cognitive optimality at the narrative level. For instance, in prior studies, equal proportions of intuitive and counterintuitive concepts were used in each story (Barrett \& Nyhof, 2001; Boyer \& Ramble, 2001). The research reported in this article was designed explicitly to test the hypotheses that MCI narratives are more memorable, and that they therefore enjoy more cultural success than narratives fitting alternative cognitive templates. Study 1 examined whether MCI idea lists enjoy better recall after a 1-week delay than all-intuitive or maximally intuitive ones. In Study 2, we examined whether this memory bias explains the cultural success of folktales in the Grimm Brothers collection, such that MCI folktales are more likely to attain sustained popularity than those that do not. In both studies we expected the relation between minimal counterintuitiveness and cognitive or cultural success to fit a nonlinear inverted $U$-shaped curve.

\section{Study 1: Memory for MCI lists}

\subsection{Overview}

We conducted an experiment to examine the memorability of MCI idea lists. To create lists with different proportions of counterintuitive ideas, we first created individual intuitive and counterintuitive items. The latter were created by transferring a property from its intuitive domain to a novel domain (e.g., thirsty door). Recall was measured after a 3-min delay, and after a 1-week delay. This latter measure was the central one, as it reflects the proper role of recall in the cultural evolution of narrative structures. This study differed from previous ones in a number of important ways. One difference is of primary interest: Lists were used that varied in the relative proportion of intuitive (INT) and MCI ideas. This allowed us to test the cognitive optimality hypothesis at the level of lists of ideas, with implications for the memorability of narratives, as well as at the level of the individual ideas.

Several other methodological differences are notable as well. First, the INT and MCI ideas were rigorously matched, such that each word served as its own control. In addition, participants were told that they were in an experiment about memory, and were given a list of items to remember, without providing a story context. This served two purposes. First, this list-learning format provided as neutral a context as possible to measure recall, eliminating any effects due to participants' notions of what would be conversationally interesting to report. Second, although stories are an important part of culturally successful materials, many of these stories often begin their life as a set of discrete images and events, with little or no story structure. Our experimental format simulated the degraded informational context of nascent cultural materials. Finally, basic-level concepts were used-for example, door, cat, infant (Rosch, Mervis, Grey, Johnson, \& Boyes-Braem, 1976).

Two questions were examined: (a) At the level of individual ideas, which ones enjoy better recall: MCI ideas or their intuitive counterparts? (b) At the level of the entire list, what proportion of intuitive to MCI ideas maximizes recall of the entire list? It was expected, consistent with prior research, that individual MCI ideas would enjoy a recall advantage over intuitive 
ones. More central to this research, it was expected that at the list level, MCI lists would enjoy the highest rate of recall (compared to lists with equal proportions of intuitive and MCI ideas, those composed entirely of intuitive ideas, and those composed primarily of MCI ideas).

\subsection{Method}

\subsubsection{Generation of intuitive and counterintuitive items}

Two-word statements that represented INT and MCI items were generated. Each statement consisted of a concept and one property that modified it. INT statements were created by using a property that was appropriate to the ontological category (e.g., closing door). MCI statements were created by modifying the concept by a property that was transferred from another ontological category (e.g., thirsty door). This procedure explicitly operationalizes minimal counterintuitiveness as the transfer of a property associated with the core conceptual domains of folk physics, folk biology, and folk psychology, from an appropriate ontological category of person, animal, plant, substance, to an inappropriate one (Atran \& Norenzayan, 2004). For example, a "thirsty door" transfers a folkbiological property (thirst) from its proper category (animal or plant) to an improper category (inert object/substance).

For each INT statement, a matching MCI statement was generated (e.g., closing cat). Similarly, for each MCI statement, a matching INT statement was generated (e.g., thirsty cat). This resulted in a set of four statements that achieved a counterbalanced design, each word in each statement serving as its own control. Thus, "cat," "door," "closing," and "thirsty" were equally likely to appear in a INT item as in an MCI item. Table 1 shows examples of the statements

To ensure that the INT and MCI statements were successfully created, ten pretest participants were asked to rate the statements on a 5-point scale anchored by the labels "ordinary" and "out of the ordinary." Of those, 36 pairs of INT, and 36 pairs of MCI that received mean ratings less than 2 (for INT) or mean ratings more than 4 (for MCI) were retained (for a total of 18 items). As a second manipulation check, a separate group of 28 participants (13 men, 15 women, age $M=26$ ) who were blind to the purpose of the study rated the 18 items in four different item versions, on a 6-point scale anchored by the labels "very natural" and "very super-

Table 1

Selected examples of intuitive statements (INT) and their minimally counterintuitive (MCI) counterparts, in a counterbalanced design

\begin{tabular}{ll}
\hline INT & MCI \\
\hline 1. Closing door & Thirsty door \\
Thirsty cat & Closing cat \\
2. Four-legged table & Confused table \\
Confused student & Four-legged student \\
3. Drying coat & Mischievous coat \\
Mischievous comment & Drying comment \\
4. Clenched fist & Impatient fist \\
Impatient man & Clenched man \\
5. Sleeping dog & Contrived dog \\
Contrived parable & Sleeping parable \\
\hline
\end{tabular}


natural." MCI items were rated to be substantially less natural than INT items, $F(1,24)=$ 45.57, $p<.001$, and this effect did not interact with the counterbalanced item type, $F<1$.

\subsubsection{Participants}

One hundred eight undergraduate students in an introductory psychology class at a large midwestern American university participated in this study. Of those 108, 14 spoke English as a foreign language and, therefore, were dropped from the analysis. ${ }^{1}$ Of the remaining 94 (58 female, 36 male) participants, 82 took part in the second recall task after a 1-week delay.

\subsubsection{Experimental manipulation}

Two experimental manipulations were constructed. The first was a (within-subjects) manipulation of the intuitive content of each item: Some items were INT, others were MCI. The second was a (between-group) manipulation of the proportion of INT and MCI items. Participants were randomly assigned to one of four experimental conditions each corresponding to a different list structure:

1. Entirely intuitive (18 INT items, 0 MCI items).

2. Minimally counterintuitive (13 INT items, 5 MCI items).

3. Equal frequencies (9 INT items, 9 MCI items).

4. Mostly counterintuitive (5 INT items, $13 \mathrm{MCI}$ items).

In the "equal frequencies" condition, four different narrative lists were created, each of which included one of the four possible concept + modifier combinations: for example, closing door (INT), thirsty cat (INT), thirsty door (MCI), closing cat (MCI). Thus the "equal frequencies" condition controlled for any possible baseline differences in the memorability of these concepts and modifiers. For the other three experimental conditions, two different lists were created, by randomly selecting a subset of the INT and MCI items from those used in the "equal frequencies" condition.

\subsubsection{Procedure}

Participants were tested in a classroom setting, using questionnaires. They were timed for each part of the study. The first page of the questionnaire contained instructions: They were asked to study the items on the list, on the next page to recall them later. After spending 5 min studying the list, the experimenter signaled them to turn to the following page, on which they spent 3 min completing a distractor task. Then they turned the page and listed as many of the items as they could remember on a blank sheet. They were given 5 min to complete this recall task. After exactly 1 week, the same experimenter gave the same participants a surprise recall task. In $5 \mathrm{~min}$, they wrote down on a blank sheet of paper as many of the statements as they could recall from the week before.

\subsubsection{Dependent measures}

Three dependent variables were assessed. Free recall after a 3-min distractor task, delayed free recall again after a 1-week distractor task, and memory degradation (immediate recall minus delayed recall). Recall was measured as follows: Participants received a score of 2 for recalling the two-word statement fully, a score of 1 for recalling only one of the two words in the 
statement, and a score of 0 for failing to recall any part of the statement. Remembering words or statements that did not appear on the list was considered failure to recall and given a score of 0 .

\subsection{Results}

Based on this formula, a percentage measure of recalled items was computed in each condition. In addition, an INT percentage score was calculated by adding up the scores for each statement, and dividing the sum by the number of INT statements that appeared on the given list. Similarly, an overall MCI percentage score was calculated for each participant (except in the entirely intuitive condition). Thus, for both immediate and delayed recall, each participant had an overall recall score, an INT score, and an MCI score. Comparisons of overall recall across the four experimental conditions, as well as the memory degradation (immediate minus delayed) measure tested the novel hypothesis that MCI lists are more memorable. Direct comparison between INT and MCI indexes within each condition tested the hypothesis (supported in previous research) that MCI elements are more memorable.

\subsubsection{Immediate recall}

Fig. 1 presents the results for overall recall, broken down according to experimental condition. Across the four experimental conditions, a linear contrast indicated that overall recall levels increased as the proportion of counterintuitive elements decreased, the differences approaching significance, $t(90)=1.60, p=.11$. The three experimental conditions containing counterintuitives did not differ from one another, all $t<1$. As to specific items, contrary to previous findings, a paired-samples $t$ test revealed a recall advantage associated with INT items over MCI ones, $M=62.82(S D=23.18)$ versus $M=53.74(S D=24.10)$, respectively; $t(68)=$ $3.49, p<.001$. (These analyses exclude participants in the entirely intuitive condition.) This

Immediate Recall

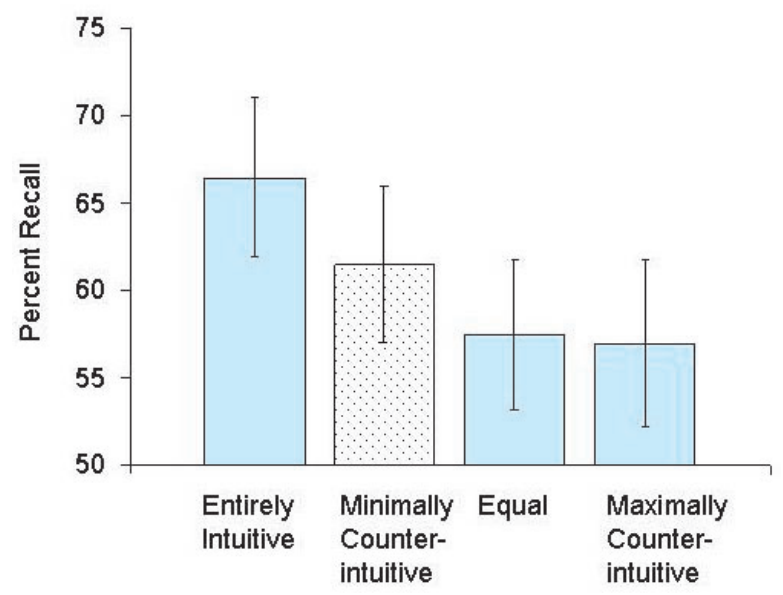

Fig. 1. Immediate recall by proportion of intuitive and MCI ideas. 
mean difference was observed within all three conditions containing counterintuitive elements $(p<.05)$.

\subsubsection{Delayed 1-week recall}

Delayed recall is the central focus of this study, because it plays the primary role in cultural transmission. Fig. 2 reveals that there were effects of the experimental manipulation on overall delayed recall, ${ }^{2}$ and these effects are consistent with the hypothesized superiority of lists that fit an MCI template and the hypothesized curvilinear effect of counterintuitive items on recall. A planned comparison indicated that recall was higher in the MCI condition compared to the combined mean in the other three conditions, $t(78)=1.88, p=.05$.

As one might expect, there was a massive overall memory degradation from the immediate recall context $(M=60.67, S D=22.03)$ to delayed recall after a week $(M=25.20, S D=16.70)$, $t(81)=18.97, p<.001$. Nevertheless, the memory advantage associated with intuitive elements persisted, $M=28.38(S D=20.43)$ versus $M=22.39(S D=17.41), t(59)=2.93, p<.005$ (except there was no effect within the mostly counterintuitive condition, $t<1$ ).

\subsubsection{Memory degradation}

Subtracting delayed recall from immediate recall produced a measure of memory degradation. This is yet another measure of the cultural resilience of cognitive templates. Such a measure is conceptually distinct from delayed recall and captures the extent to which a cognitive structure, once encoded, degrades for a given period of time. As Figure 3 indicates, the MCI list had the least memory degradation compared to the combined mean of the three other conditions, $t(78)=1.93, p=.06$. No overall differences were found between intuitive and MCI items across the three conditions, $t(59)=1.18, p=n s$. Nor were there differences in each condition $($ all $p s=n s)$.

\section{Delayed Recall-1 Week}

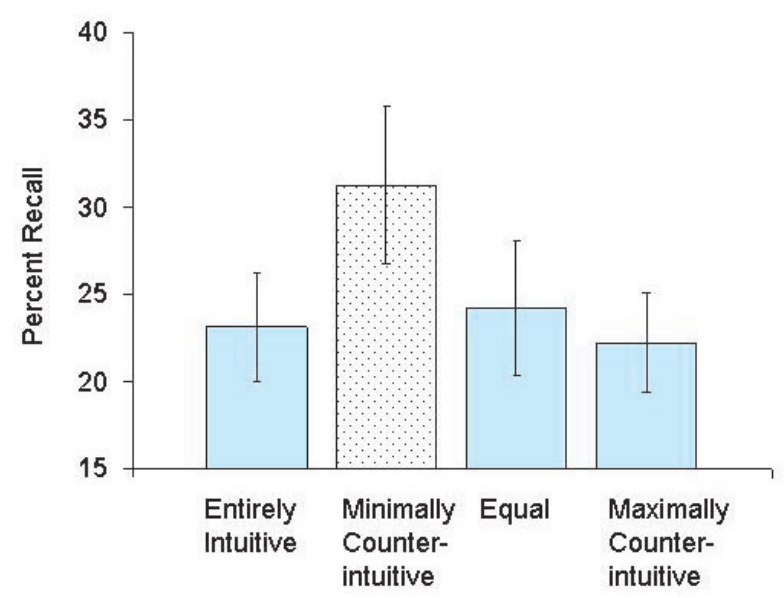

Fig. 2. Delayed 1-week recall by proportion of intuitive and MCI ideas. 


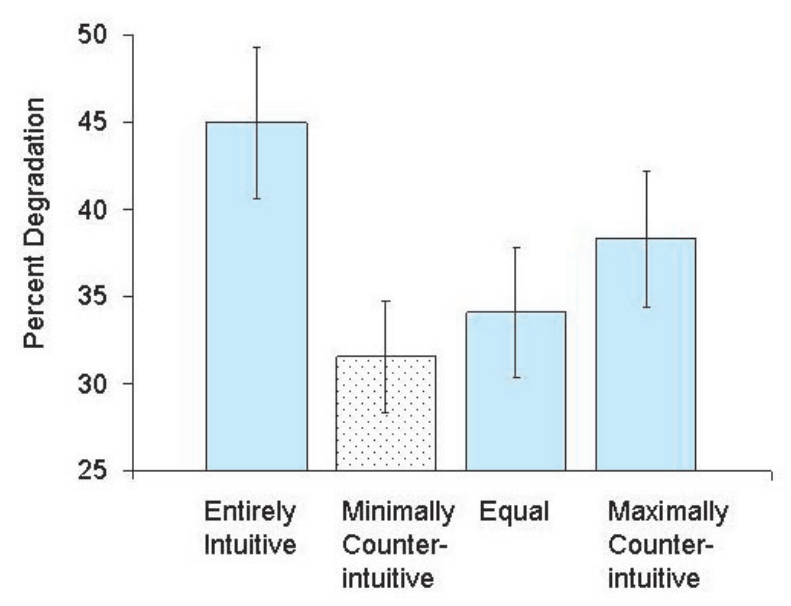

Fig. 3. Memory degradation (immediate minus delayed recall).

\subsection{Discussion}

Consistent with the central hypothesis in this article, the results confirmed that cognitive optimality at the level of lists of ideas is important. Although immediate recall, if anything, was a linear function of proportion of counterintuitives and did not favor MCI lists, delayed recall did. MCI lists were the most cognitively resilient. Thus, although minimal counterintuitiveness may not always enhance the memorability of specific ideas, at a different level of analysis it does appear to enhance the memorability of lists of ideas. This may provide the recipe for a successful cultural knowledge structure; indeed it is the cognitive template that characterizes many popular narratives, including religious accounts, myths, fables, and folktales.

As to ideas, a complex pattern of recall emerged. Unlike the findings of Barrett and Nyhof (2001) and Boyer and Ramble (2001), but consistent with the early classic experiments of Bartlett (1932), intuitive ideas showed better recall rates than MCI ones. This was the case immediately, as well as after a 1-week delay. The only exception to this pattern was when counterintuitives made up the majority of items, in which case there were no differences in delayed recall rates. Also there was no advantage for intuitive items in memory degradation. Because the two kinds of elements were rigorously matched (i.e., each word in each element was equally likely to occur in intuitive and MCI items), this recall advantage is clearly attributable to intuitiveness, rather than to other variables related to word recall. Subsequently, we have replicated this finding showing a long-term recall advantage for intuitive items (after 1 week and also after 3 months), using more stringent criteria for counterintuitiveness, with American college students, as well as Itza' Maya villagers in the Mexican Yucatan (for summaries, see Atran \& Norenzayan, 2004; Norenzayan \& Atran, 2004).

How can we account for the superiority of intuitive items in recall? There are two important methodological differences between our study and the prior experiments of Barrett and Nyhof (2001) and Boyer and Ramble (2001). In those experiments, participants were given descrip- 
tions of counterintuitive objects or events in the abstract ("a person who was at two places at the same time"), whereas in our experiment we provided basic-level descriptions (Rosch et al., 1976) that are typically found in supernatural narratives ("talking frog").

Second, in earlier studies participants were primed to expect counterintuitive events (as in listening to a science fiction tale), or they were motivated to tell an interesting story (as in the serial transmission paradigm). Such contexts are indeed important vessels of cultural transmission, as in storytelling events or religious rituals. On the other hand, they have the disadvantage that they do not provide the ideal context in which the impact of recall, controlling for other psychological or social variables, can be examined. This experiment used a simple memory task that minimized the role of social or communicative factors that are related to, but distinct from, the processes of memory. In such a context in which people expect that information will conform to a natural course of events, they are likely to attend to and remember items that are consistent with intuitive assumptions.

\section{Study 2: Cultural success of MCI narratives in folktales}

\subsection{Overview}

Study 1 revealed that MCI lists enjoy a recall advantage over time. This finding has implications for the interpersonal transmission of narrative structures such as folktales and for their consequent popularity across a cultural landscape (Sperber, 1996). Memorable narrative structures are more likely to be transmitted, so they may enjoy a relative advantage in the marketplace of cultural beliefs; ultimately, they are likely to become and remain more popular. To test this hypothesis, we examined the narrative features of folktales that have had varying degrees of cultural success.

Our study focused on one of the most culturally important folktales in the Western tradition-collected by the Brothers Grimm. We empirically assessed the cultural success of each folktale, and selected 42 tales for deeper analysis; 21 were relatively successful, and 21 were demonstrably less successful. Two trained raters, unaware of our hypotheses, read each tale and counted the number of counterintuitive elements in each folktale. In addition, 65 university students read these folktales and rated them on a number of characteristics, including memorability and ease of transmission. These procedures allowed us not only to test the hypothesis that MCI tales are more likely to be culturally successful, but also to examine whether perceived memorability mediates the relation between minimal counterintuitiveness and cultural success.

\subsection{Method}

As a source, we used Manheim's (1857/1977) English translation of the 1857 edition of the Grimm Brothers collection (published originally in German). This collection contains 200 folktales, some of which are currently very well known by many European peoples (e.g., "Rapunzel," "Hansel and Gretel," "Cinderella") and many more stories that are relatively unknown (e.g., "Brother Scamp," "The Donkey Lettuce, " "A Good Stroke of Business"). 


\subsubsection{Culturally successful and unsuccessful folktales}

To obtain a measure of cultural popularity, we conducted 400 searches of the World Wide Web, using the search engine Google. Each search included as keywords the exact title of each folktale, along with the word "Grimm" (e.g., "Hefty Hans" + Grimm). Half the searches used original German titles, and half used English translations of the titles (when necessary, we searched for multiple English-translated titles). We added the word "Grimm" to all title searches to ensure that the content of found Web sites was relevant to the folktales of interest. For each search, we counted the number of Web page "hits" as a rough indicator of cultural popularity. The English and German searches yielded convergent but not identical results, $r(41)=.49, p=.001$.

Not surprisingly, there was great variability in the number of Web page hits across the 200 different folktales (ranging from 34,430 for "Cinderella" to 12 for "The Ditmarsh Tale of Lies"). We used these numerical results as a basis for selecting a sample of culturally successful tales. We identified a list of the 20 folktales whose German titles produced the most hits, and another list of the 20 tales whose English titles produced the most hits. Together, these lists were composed of 30 different folktales. We eliminated from further consideration 9 folktales whose titles consisted simply of one or two common words (e.g., "The Moon"), as these tales were especially likely to have produced spurious hits. Twenty-one tales remained, and these defined our sample of culturally successful folktales (see Table 2); the mean number of hits across these 21 tales was 8,404 .

To select a comparison group of culturally unsuccessful folktales, we excluded from consideration any tale that was among the 50 most popular identified by either the German-language or English-language Google searches. This exclusion criterion left 126 relatively unsuccessful folktales. From this set, we selected 21 tales (see Table 2), attempting to ensure that they were of approximately the same length as those in the culturally successful sample (for both sam-

Table 2

Culturally successful and unsuccessful folktales

Culturally successful ( $N=21)$ : “The Frog King” ("Iron Henry”) (1), "Little Brother and Little Sister" (11), "Rapunzel" (12), "Hansel and Gretel" (15), "The Fisherman and his Wife" (19), "The Brave Little Tailor" (20), "Ashputtle" (“Cinderella") (21), "Mother Holle” (24), "Little Red Cap" ("Little Red Riding Hood”) (26), "The Musicians of Bremen" (27), "The Devil with the Three Golden Hairs" (29), "Brier Rose" ("Sleeping Beauty") (50), "King Thrushbeard" (52), "Snow White” (53), "Rumpelstiltskin" (55), "Thousandfurs" (65), "Jorinde and Joringel" (69), "Hans in Luck" (83), "The Lilting, Leaping Lark" ("Beauty and the Beast") (88), "The Goose Girl” (89), "Snow White and Rose Red" (161).

Culturally unsuccessful ( $N=21)$ : “A Good Stroke of Business" (7), “The Girl Without Hands" (31), "The Magic Table, The Gold Donkey, and the Cudgel in the Sack" (36), "The Knapsack, the Hat, and the Horn" (54), "Frederick and Liza-Kate" (59), "Farmer Little" (61), "Six Who Made Their Way in the World" (71), "The Carnation" (76), "Brother Scamp" (81), "The Golden Children" (85), "The King of the Golden Mountain" (92), "The Spirit in the Bottle" (99), "Bearskin" (101), "Hans My Hedgehog" (108), "The Jew in the Brambles" (110), "The Prince Who Feared Nothing" (121), "The Donkey Lettuce" (122), "Faithful Ferdinand and Faithless Ferdinand" (126), "Hefty Hans" (166), "The Poor Boy in the Grave" (185), "Maid Maleen" (198).

Note. Included in brackets following each title is the order number where each folktale appeared in the 1857 Grimm Brothers collection. 
ples, the mean length was slightly greater than four pages). Aside from this attempt to match on length, selection was random. The mean number of Google hits across these 21 unsuccessful tales was 148.

\subsubsection{Counterintuitive and bizarre narrative elements}

Two trained raters, unaware of our hypotheses, judged the number of counterintuitive elements in each of the 42 folktales. Raters counted as counterintuitive any narrative element that defies intuitive assumptions about the ontological properties of the everyday world, such as the categorical and relational properties that people in all cultures appear to spontaneously ascribe to intentional agents (folk psychology), biological kinds (folk biology) and inert bodies (folk physics; see Atran \& Norenzayan, 2004; Boyer, 2003). Recurring counterintuitive characters, objects, or events were counted as single counterintuitive elements (e.g., a talking mirror may appear multiple times in the same story, but counts as a single counterintuitive element). The same raters also judged the number of bizarre elements. Narrative elements were counted as bizarre if they were fancifully out-of-the-ordinary, but did not fit the strict definition of counterintuitive as an ontological violation (e.g., a house made of gingerbread).

Across all 42 tales, interrater reliability was very high for counterintuitive elements (Cronbach's $\alpha=.92$ ) and somewhat less high for bizarre elements $(\alpha=.70)$. For subsequent analyses, indexes of counterintuitive and bizarre elements were created by calculating mean judgments across the two raters.

\subsubsection{Memorability and other psychological variables}

Sixty-five students (age $M=20,52$ women and 13 men, 14 European Canadian, 36 East Asian Canadian, and 15 other) at the University of British Columbia read six folktales apiece. Each set of six was selected from the total sample of 42 folktales. Each rater read a different combination of tales. Consequently, each folktale was read and rated by at least seven different raters (21 tales were rated 10 times, 16 were rated 9 times, 1 was rated 8 times, and 4 tales were rated 7 times).

After reading each tale, raters completed a questionnaire assessing their impressions. They first indicated simply whether they were familiar with the folktale or not. Participants then rated each tale on a number of attributes on 7-point scales (anchored by endpoints labeled Strongly Disagree to Strongly Agree). One rating assessed understandability ("This story was easy to understand"). Another rating assessed ease of transmission ("If I wanted to tell this story to other people, I could do so quickly and easily"). Two items assessed perceived interest value ("This story was interesting," and "Children would find this story interesting"). Two items assessed memorability ("Right now if someone asked me to tell them the story that I just read, I think I could recall all or most of the critical elements of the story," and "One month from now if someone asked me to tell them the story that I just read, I think I could recall all or most of the critical elements of the story"). Two items assessed likelihood of transmission to peers ("I would talk about this story with my friends," and "If I told a friend this story, he or she would tell it to other friends"). Two more items assessed likelihood of transmission to children ("I would talk about this story with a 7-year-old child," and "If I told a 7-year-old this story, he or she would tell it to other 7-year-olds"). Finally, participants indicated their agreement with the statement "This story contains a moral lesson." 
Raters then completed one additional measure designed to assess the communicability of each folktale. They were asked to consider a situation in which they were given an opportunity to tell only a few of the stories to others, and they then ranked the stories to indicate their relative motivation to communicate each story to others. (A ranking of 1 was given to the story they deemed most highly communicable; higher values indicate lower communicability).

Data were collapsed across raters so as to treat folktale as the unit of analysis. Thus, each datum was composed of the mean of seven or more independent ratings of each story. Four constructs were assessed by two items apiece, and results on these items were combined to create two-item composite indexes (for interest value, memorability, transmission to peers, and transmission to children; Cronbach's $\alpha$.67).

\subsection{Results}

\subsubsection{Manipulation check of cultural success variable}

University students' judgments of folktale familiarity provide a check on the Web-search-based categorization of tales as successful or unsuccessful. Reassuringly, these raters were more familiar with folktales categorized as successful, $t(40)=4.46, p<.001$. On average, culturally successful stories were familiar to $41 \%$ of raters, whereas unsuccessful stories were familiar to only $5 \%$ of raters. In subsequent analyses we treat cultural success as a dichotomous variable.

\subsubsection{Are MCI folktales more culturally successful?}

The hypothesized relation between counterintuitive elements and cultural success is nonlinear. Although there is no necessary implication for differences in mean numbers of counterintuitive elements (and no meaningful mean differences were observed), the hypothesis predicts that there will be different shapes to the distributions of counterintuitive elements within the samples of successful and unsuccessful folktales: Compared to unsuccessful folktales, the distribution within the sample of successful tales should be more clearly unimodal, and variability around that central tendency should be relatively low. The two frequency distributions are displayed in Figure 4. As hypothesized, variability within the successful sample $(S D=1.65)$ was lower than within the unsuccessful sample $(S D=2.19), F(1,20)=3.92, p=$ .05 (using Levene's test for equality of variances). Visual inspection reveals that, among culturally unsuccessful tales, the distribution is relatively flat and there is no single modal number of counterintuitive elements. In contrast, among culturally successful tales, there is a clear mode: The majority of these tales had a counterintuitive score between 2 and 3, inclusive. Using this range ( 2 to 3 ) to define a set of MCI folktales, it is revealed that $76.5 \%$ of MCI tales are in the culturally successful sample. In contrast, among stories with few or no counterintuitive elements (scores $<2$ ), only $30 \%$ were culturally successful. Similarly, among stories with excessive numbers of counterintuitive elements (scores $>3$ ), only $33 \%$ were culturally successful. Thus MCI folktales were more likely to be culturally successful, $\chi^{2}(N=42)=8.00, p=.005$. Minimal counterintuitiveness correctly predicted cultural success of folktales $71.4 \%$ of the time.

Whereas the two sample distributions clearly differ in terms of counterintuitive elements, investigation of bizarre elements revealed no apparent differences. Within both samples, the 


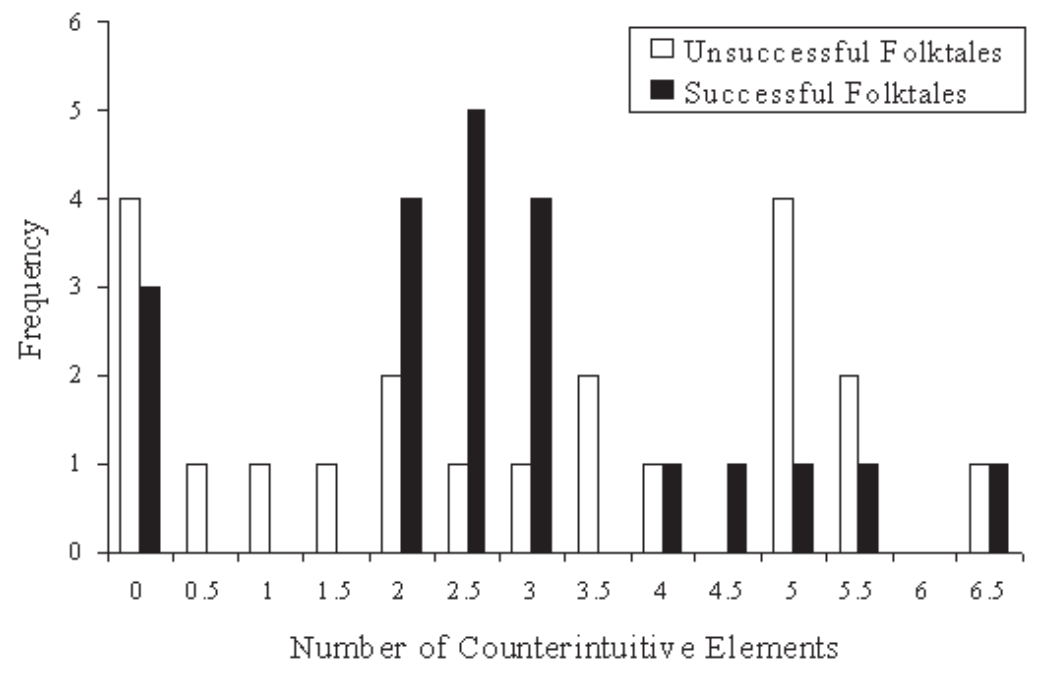

Fig. 4. Frequency distribution of counterintuitive elements contained in samples of culturally successful and unsuccessful folktales.

number of bizarre elements in each story was relatively low $(M \mathrm{~s}=1.17$ and 1.36 for successful and unsuccessful tales), and there were no meaningful differences in the shape of the distributions (e.g., variability was very similar; $S D s=1.10$ and 0.98 for successful and unsuccessful folktales, respectively). Thus, although the results indicate that cultural success is predicted by the number of counterintuitive elements, success is not predicted by unusual narrative elements more broadly.

\subsubsection{Are MCI folktales psychologically different?}

The preceding set of results provided an empirical criterion for defining a folktale as MCI or not, which then allowed us to test whether MCI folktales are perceived to be more memorable, and more psychologically appealing in other ways as well. We divided the total sample of 42 folktales into two categories: those that were MCI and those that were not. Folktales were counted as MCI if the mean number of counterintuitive elements fell between 2 and 3, inclusive $(\mathrm{N}=17)$. All other folktales (with scores less than 2 or greater than 3 ) were placed in the comparison category $(\mathrm{N}=25)$. Table 3 summarizes mean ratings on memorability and other psychological variables and also provides inferential statistics pertaining to mean differences.

Consistent with the hypothesis, these results reveal that MCI folktales were perceived to be more memorable. They were also perceived to be more understandable and easier to transmit than folktales containing either too few or too many counterintuitive elements. No differences were found for interest value, transmission value, and moral lesson. The difference approached significance for communicability (see Table 3).

\subsubsection{Are culturally successful folktales psychologically different?}

These methods also allowed us to test whether culturally successful folktales also differ from unsuccessful ones on ratings of memorability and other psychological variables 
Table 3

Mean ratings on psychological variables, as a function of whether a folktale is minimally counterintuitive or not

\begin{tabular}{lllll}
\hline & \multicolumn{2}{l}{ Minimally Counterintuitive } & & \\
\cline { 2 - 5 } & Yes & No & $t$ & \\
\hline Memorability & 4.89 & 4.37 & 2.42 & .02 \\
Understandability & 6.24 & 5.74 & 3.19 & .003 \\
Ease of transmission & 5.46 & 5.02 & 2.02 & .05 \\
Interest value & 5.03 & 4.83 & 0.97 & .34 \\
Transmission to peers & 2.80 & 2.68 & 0.80 & .43 \\
Transmission to children & 4.05 & 3.81 & 0.95 & .35 \\
Communicability (Rank) & 3.27 & 3.71 & 1.58 & .12 \\
Moral lesson & 4.59 & 4.43 & 0.50 & .62 \\
\hline
\end{tabular}

(Table 4). Results revealed mean differences on most rated characteristics, indicated that culturally successful folktales were overall psychologically privileged. Culturally successful tales were judged to be relatively more memorable, understandable, interesting, and easier to transmit. In addition, all three indexes assessing likelihood of transmission revealed that compared to demonstrably unsuccessful tales, culturally successful folktales are more likely to be communicated to others.

\subsubsection{Does memorability mediate the relation between minimal counterintuitiveness and cultural success?}

The preceding results are consistent with the following chain of reasoning: MCI folktales are more memorable and easier to understand and transmit to others. As a result of their superior memorability and understandability (but not necessarily other characteristics), they are more likely to become culturally popular. If indeed a folktale's memorability mediates the relation between its status as an MCI narrative and cultural success, then the observed relation be-

Table 4

Mean ratings on psychological variables, as a function of whether a folktale is culturally successful or not

\begin{tabular}{lllll}
\hline & \multicolumn{2}{l}{ Culturally Successful } & & \\
\cline { 2 - 4 } & Yes & No & $t$ & $p$ \\
\hline Memorability & 5.03 & 4.13 & 4.95 & $<.001$ \\
Understandability & 6.29 & 5.60 & 4.72 & $<.001$ \\
Ease of transmission & 5.61 & 4.78 & 4.30 & $<.001$ \\
Interest value & 5.20 & 4.61 & 3.00 & .005 \\
Transmission to peers & 2.83 & 2.63 & 1.46 & .15 \\
Transmission to children & 4.41 & 3.40 & 4.97 & $<.001$ \\
Communicability (Rank) & 3.01 & 4.04 & 4.41 & 1.67 \\
Moral lesson & 4.76 & 4.23 & .001 \\
\hline
\end{tabular}


tween minimal counterintuitiveness and cultural success is likely to be reduced or eliminated when controlling for the alleged cognitive mediator.

To investigate this possibility, folktales were categorized as either MCI or not (coded as 1 and 0 , respectively) and as successful or not (coded as 1 and 0 , respectively), according to the categorization criteria described previously. The zero-order correlation between these two variables was $\varphi=.44, p=.005$. The two memorability items, understandability and ease of transmission, were strongly intercorrelated and showed very high internal reliability $(\alpha=.94)$; as a result, they were combined to reflect a composite "cognitive facility" variable. Results from a logistic regression analysis revealed that, when cognitive facility was included along with minimal counterintuitiveness as predictors of cultural success, the predictive impact of minimal counterintuitiveness was reduced from $\beta=1.93$, Wald $\chi^{2}(N=42)=7.31, p=.007$, to $\beta$ $=1.45$, Wald $\chi^{2}(N=42)=2.60, p=.12$. The cognitive facility variable continued to exert an impact on cultural success, $\beta=3.22$, Wald $\chi^{2}(N=42)=9.03, p=.003$. This result suggests that the cognitive facility variable partially (but not completely) mediated the relation between a folktale's status as MCI and its eventual cultural success.

\subsection{Discussion}

As hypothesized - and consistent with previous findings-MCI folktales were rated as especially memorable. They also were rated as more understandable, and easier to transmit to others. These cognitive features can have cultural consequences as well. The cultural success of a folktale - defined by its popularity — was predicted by the number of counterintuitive elements. Success was not predicted by the number of intuitive but bizarre elements. Moreover, there was evidence that the cognitive features of a folktale-specifically its memorability, understandability, and ease of transmission-mediated the relation between minimal counterintuitiveness and cultural success. Although the inferences we can draw from the mediational analysis are limited by the small sample size and the self-report-based measures, it is reassuring that these measures appropriately distinguished the cognitive templates of folktales from their cultural success. Whereas the minimal counterintuitiveness of a folktale predicted specific cognitive characteristics related to memorability, understandability, and ease of transmission, the cultural popularity of a folktale predicted a general favorability on most of the assessed psychological measures. MCI narrative structures are not only more memorable, as suggested by the recall evidence in Study 1, but this memorability also accounts in part for their cultural success over time.

\section{General discussion}

Religious and supernatural narratives, including partly counterintuitive narratives that are found in myths, legends, and folktales are extraordinarily resilient aspects of human cultures around the world. Most of these narratives have been transmitted orally through generations and have come to shape beliefs, values, and behaviors of people everywhere. What accounts for the persistence of such narratives? No doubt many cognitive (Rubin, 1995), emotional (Heath, Bell, \& Sternberg, 2001), and ecological (Anderson \& Schooler, 1991; Berger \& 
Heath, 2005) variables likely contribute to the cultural stability of such widespread belief structures. In this article we examined one very important cognitive factor-the memorability advantage that MCI narratives enjoy in the minds of cultural members. We found that MCI lists that contain only a few counterintuitive elements degrade less over time and produce superior recall in the long term (but not in the short term) over other templates that are either entirely intuitive or excessively counterintuitive (Study 1). This cognitive advantage also selectively predicts the cultural success of one type of culturally important narrative-folktales (Study 2).

Elaborating on early insights by Sperber and others (1975; 1996, Lawson \& McCauley, 1990), supernatural beliefs are being examined from a natural science perspective within what has come to be known as the cognitivist program of religion (e.g., Atran, 2002; Barrett, 2000, 2004; Boyer, 1994b, 2001, 2003; Lawson \& McCauley,1990; Pyysiäinen \& Antonnen, 2002). In recent years, growing research has been examining the cognitive and communicative processes that shape and constrain cultural and religious beliefs (e.g., Barrett \& Nyhof, 2001; Boyer \& Ramble, 2001). Whereas previous studies focused primarily on the cultural selection of discrete supernatural concepts, here we examined the cultural selection of lists of ideas and narratives that contain counterintuitive elements and often act as a coherent unit of transmission across minds.

We propose that MCI narratives are culturally successful partly because they enjoy a stronger cognitive advantage in recall than other narrative templates. Although the specific computational properties of this memory advantage remain an open question, we offer two possible cognitive explanations for future empirical investigation. First, the literature on the psychology of memory has emphasized that psychological factors that encourage deeper and more elaborate processing of the information produce superior recall and greater number and quality of inferences (e.g., Craik \& Lockhart, 1972; Craik \& Tulving, 1975). MCI narratives demand a greater cognitive effort in connecting the new material with background knowledge, as well as the minority counterintuitive elements with the majority intuitive elements, spurring deeper and more elaborate processing. For example, work by Kintsch and his colleagues (McNamara \& Kintsch, 1996; McNamara, Kintsch, Songer, \& Kintsch, 1996) examining text comprehension found that texts with coherence gaps lead to superior recall than texts with no gaps for learners with adequate background knowledge of the topic. A reverse pattern is found for learners with low background knowledge. Thus, texts that elicit some degree of active inferencing on the part of the knowledgeable learner can help memory. However, as in the case of our findings and other research (e.g., Boyer \& Ramble, 2001), this effect is curvilinear-too many coherence gaps are detrimental to memory and learning (Kintsch, 1994).

Second, MCI narratives may enjoy a memory advantage because they generate the greatest number of inferences with the least cognitive effort, as predicted by relevance theory (Sperber $\&$ Wilson, 1986). That is, this cognitive template maximizes relevance by deviating from ordinary expectations systematically but not excessively. These deviations involve evocative minimal counterintuitions that are "relevant mysteries" (Sperber, 1996, p. 73). They are closely connected to background knowledge, but do not admit to a final interpretation. As a result they are attention arresting and inferentially rich, and therefore encourage further cognitive processing and multiple interpretations over time that facilitate the cognitive stabilization of narratives. But only if the ensuing impossible worlds remain largely connected to the everyday 
world can supernatural narratives be mentally represented, rehearsed, and transmitted (Atran \& Sperber, 1991; Barrett, 2000; Boyer, 2003; Sperber, 1975).

Regardless of which processes best explain the memory advantage, examining cognitive optimality at the level of lists and narratives is critical. If cognitive optimality were operating at the level of distinct concepts only, and if the lists and narratives in which they are embedded did not have any cognitive effect, we would expect that the cognitive or cultural success of a narrative would increase as a linear function of the proportion of MCI concepts. However this was not the case. In Study 1, only lists with a few counterintuitive ideas facilitated long-term recall. Similarly in Study 2, only folktales with a few counterintuitive elements facilitated cultural success. In both studies, the effect of the number of counterintuitive elements on cognitive or cultural success was a curvilinear function.

Counterintuitiveness, whether examined at the level of concepts or narratives, is distinct from bizarre conceptual features that otherwise do not violate core expectancies rooted in core domain-specific theories. Although Study 1 did not examine bizarreness as distinct from intuitive violations, evidence for this was found in Study 2, in which the number of bizarre elements had no predictive power in accounting for the cultural success of folktales, whereas number of counterintuitive elements did. This is consistent with prior findings indicating that intuitive violations and strangeness are two distinct processes and only the former reliably affects the recall of supernatural ideas (e.g., Boyer \& Ramble, 2001).

Our findings were robust, observable whether in a controlled laboratory context, or in the content analysis of naturally occurring cultural narratives. However, one limitation regarding these studies needs mention. In both studies the samples were representative of Western culture. It would be important to examine the extent to which MCI narratives are also found to favor cultural success in non-Western cultural contexts. In addition to laboratory experiments, the rich traditions of folktales and myths of diverse cultures, such as Hindu Vedic tales, or the Mayan Popul Vuh, could be examined in search of the MCI cognitive template described in this article. Such cross-cultural extensions also could shed light on culture-specific cognitive structures that no doubt contribute to their cultural success (e.g., Brewer, 1985; Kintsch \& Greene, 1978).

Finally, we emphasize that we do not claim that human memory was "designed" to favor MCI narratives such as the ones we observe in religious and mythical traditions. Quite the contrary, such narratives were most likely culturally selected because they successfully exploited the already existing cognitive architecture of the human memory system, which was naturally selected to solve adaptive problems that were quite unrelated to the propagation and cultural stabilization of counterintuitive narratives. Thus, memorability is necessary, but not sufficient in the cultural selection of such narratives.

Once MCI narratives pass the test of long-term memorability, the supernatural elements in such narratives are further culturally exploited to play a motivational function. Supernatural elements externalize and emotionally relieve core existential human problems, including death, deception, meaninglessness, and other problems that are factually and rationally intractable (Atran \& Norenzayan, 2004; Norenzayan \& Atran, 2004). For example, there is evidence that supernatural beliefs relieve the human awareness of mortality. Controlled motivational experiments show that mortality awareness causes stronger religiosity, heightened belief in the divine, and more faith in the efficacy of supernatural interventions (Norenzayan \& Hansen, 
2006). Moreover, this effect emerges even when the supernatural agents are culturally alien, such as Christians who are faced with belief in a supernatural Buddha or in shamanic spirits. Cognitive factors alone cannot explain the cultural success of supernatural ideas and narratives. It is the convergence of cognitive and motivational vectors that determine the overwhelming presence and resilience of supernatural narratives in cultures around the world.

\section{Notes}

1. Including those participants who spoke English as a foreign language produced very similar pattern of results, although the statistical tests yielded marginal effects in some instances.

2. An alternative method of analyzing the effect of experimental condition of varying proportions of counterintuitives would be to assess recall of intuitive items only. Such an analysis was conducted and yielded the same pattern of results for the planned comparison of the mean in the MCI narrative condition against the combined mean of the three other conditions, $t(78)=1.80, p=.07$.

\section{Acknowledgments}

Ara Norenzayan, Jason Faulkner, \& Mark Schaller, Department of Psychology, University of British Columbia, Vancouver, Canada. Scott Atran, Institut Jean Nicod, Paris, France and University of Michigan, Ann Arbor, USA. Thanks to Eshanie Kirtisinghe, Brian Malley, and Sheffield Yeung for their contributions to conducting the studies reported in this article. This research has been supported by a Social Sciences and Humanities Research Council of Canada grant to the first author (Grant No. 410-2004-0197).

\section{References}

Alba, J. W., \& Hasher, L. (1983). Is memory schematic? Psychological Bulletin, 93, 203-231.

Anderson, J. R., \& Schooler, L. J. (1991). Reflections of the environment in memory. Psychological Science, 2 , 396-408.

Atran, S. (1998). Folkbiology and the anthropology of science: Cognitive universals and cultural particulars. Behavioral and Brain Sciences, 21, 547-609.

Atran, S. (2002). In Gods we trust: The evolutionary landscape of religion. Oxford, England: Oxford University Press.

Atran, S., \& Norenzayan, A. (2004). Religion's evolutionary landscape: Counterintuition, commitment, compassion, communion. Behavioral and Brain Sciences, 27, 713-770.

Atran, S., \& Sperber, D. (1991). Learning without teaching: Its place in culture. In L. T. Landsmann (Ed.), Culture, schooling, and psychological development (pp. 39-55). Norwood, NJ: Ablex.

Avis, J., \& Harris, P. L. (1991). Belief-desire reasoning among Baka children. Child Development, 62, $460-467$.

Baillargeon, R. (1998). Infants' understanding of the physical world. In M. Sabourin, F. Craik, \& M. Robert (Eds.), Advances in psychological science (Vol. 2, pp. 503-509). London: Psychology Press.

Barrett, J. L. (2000). Exploring the natural foundations of religion. Trends in Cognitive Science 4, 29-34. 
Barrett, J, L. (2004). Why would anyone believe in God? Lanham, MD: AltaMira.

Barrett, J. L., \& Keil, F. (1996). Conceptualizing a non-natural entity: Anthropomorphism in God concepts. Cognitive Psychology, 31, 219-247.

Barrett, J. L., \& Nyhof, M. A. (2001). Spreading nonnatural concepts: The role of intuitive conceptual structures in memory and transmission of cultural materials. Journal of Cognition and Culture, 1, 69-100.

Bartlett, F. A. (1932). Remembering: A study in experimental psychology. Cambridge: Cambridge University Press.

Berger, J., \& Heath, C. (2005). Idea habitats: How the prevalence of environmental cues influences the success of ideas. Cognitive Science, 29, 195-221.

Berlin, B. (1992). Ethnobiological classification: Principles of categorization of plants and animals in traditional societies. Princeton, NJ: Princeton University Press.

Berlin, B., Breedlove, D., \& Raven, P. (1973). General principles of classification and nomenclature in folk biology. American Anthropologist, 74, 214-242.

Bower, G. H., Black, J. B., \& Turner, T. J. (1979). Scripts in memory for text. Cognitive Psychology, 11, 177-220.

Boyer, P. (1992). Explaining religious ideas: Outline of a cognitive approach. Numen, 39, 27-57.

Boyer, P. (1994a). Cognitive constraints on cultural representations: Natural ontologies and religious ideas. In L. A. Hirschfeld \& S. A. Gelman (Eds.), Mapping the mind: Domain specificity in cognition and culture (pp. 391-411). New York: Cambridge University Press.

Boyer, P. (1994b). The naturalness of religious ideas. Berkeley: University of California Press.

Boyer, P. (2001). Religion explained: The evolutionary origins of religious thought. New York: Basic Books.

Boyer, P. (2003). Religious thought and behaviour as by-products of brain function. Trends in Cognitive Sciences, 7 , $119-124$.

Boyer, P., \& Ramble, C. (2001) Cognitive templates for religious concepts: Cross-cultural evidence for recall of counter-intuitive representations. Cognitive Science, 25, 535-564.

Brewer, W. F. (1985). The story schema: Universal and culture-specific properties. In D. R. Olson, N. Torrance, \& A. Hildyard (Eds.), Literacy, language, and learning. Cambridge: Cambridge University Press.

Callahan, T., Rochat, P., Liilard, A., Claux, M., Odden, H., Itakura, S., et al. (2005). Synchrony in the onset of mental state reasoning: Evidence from five cultures. Psychological Science, 16, 378-384.

Carey, S., \& Spelke, E. (1994). Domain-specific knowledge and conceptual change. In L. A. Hirschfeld \& S. A. Gelman (Eds.), Mapping the mind: Domain specificity in cognition and cognition. Cambridge, MA: Cambridge University Press.

Craik, F. I. M., \& Lockhart, R. S. (1972). Levels of processing: A framework for memory research. Journal of Verbal Learning and Verbal Behavior, 11, 671-684.

Craik, F. I. M., \& Tulving, E. (1975). Depth of processing and the retention of words in episodic memory. Journal of Experimental Psychology: General, 104, 268-294.

Davidson, D. (1994). Recognition and recall of irrelevant and interruptive atypical actions in script-based stories. Journal of Memory and Language, 33, 757-775.

Flavell, J. H., Zhang, X.-D., Zou, H., Dong, Q., \& Qui, S. (1983). A comparison of the appearance-reality distinction in the People's Republic of China and the United States. Cognitive Psychology, 15, 459-466.

Gardner, D., Harris, P. L., Ohmoto, M., \& Hamazaki, T. (1988). Japanese children's understanding of the distinction between real and apparent emotion. International Journal of Behavioral Development, 11, 203-218.

Gelman, S. A., \& Hirschfeld, L. A. (1998). How biological is essentialism? In D. L. Medin \& S. Atran (Eds.), Folkbiology. Cambridge, MA: MIT Press.

Heath, C., Bell, C., \& Sternberg, E. (2001) Emotional selection in memes: The case of urban legends. Journal of Personality and Social Psychology, 81, 1028-1041.

Keil, F. (1989). Concepts, kinds, and cognitive development. Cambridge: Bradford Book/MIT Press.

Keil, F. (1994). The birth and nurturance of concepts by domains: The origins of concepts of living things. In L. Hirschfeld \& S. Gelman (Eds.), Mapping the mind: Domain specificity in cognition and culture (pp. 234-254). New York: Cambridge University Press.

Kelly, M. H., \& Keil, F. (1985). The more things change ... Metamorphoses and conceptual structure. Cognitive Science, 9, 403-416.

Kintsch, W. (1994). Text comprehension, memory, and learning. American Psychologist, 49, 294-303. 
Kintsch, W., \& Greene, L. (1978). The role of culture-specific schemata in the comprehension and recall of stories, Discourse Processes, 1, 1-13.

Koriat, A., Goldsmith, M., \& Pansky, A. (2000). Towards a psychology of memory accuracy. Annual Review of Psychology, 51, 481-537.

Lawson, E. T., \& McCauley, R. (1990) Rethinking religion. Cambridge, MA: Cambridge University Press.

Leslie, A. M. (1982). The perception of causality in infants. Perception, 11, 173-186.

Lillard, A. S. (1998). Ethnopsychologies: Cultural variations in theories of mind. Psychological Bulletin, 1, 3-32.

Manheim, R. (1977). Grimms' tales for young and old: The complete stories. R. Manheim (Trans.). Garden City, NY: Doubleday. (Original work published 1857)

McCabe, A., \& Peterson, C. (1990). What makes a narrative memorable? Applied Psycholinguistics, 11, 73-82.

McNamara, D. S., Kintsch, E., Songer, N., \& Kintsch, W. (1996). Are good texts always better? Interactions of text coherence, background knowledge, and levels of understanding in learning from text. Cognition and Instruction, 14, 1-43.

McNamara, D. S., \& Kintsch, W. (1996). Learning from texts: Effects of prior knowledge and text coherence. Discourse Processes, 22, 247-288.

Medin, D. L., \& Atran, S. (Eds.). (1999). Folkbiology. Cambridge, MA: MIT Press.

Norenzayan, A., \& Atran, S. (2004). Cognitive and emotional processes in the cultural transmission of natural and nonnatural beliefs. In M. Schaller \& C. Crandall (Eds.), The psychological foundations of culture. (pp. 149-169). Mahwah, NJ: Lawrence Erlbaum Associates, Inc.

Norenzayan, A., \& Atran, S. (2005). [Recall of intuitive and counterintuitive ideas in two cultures]. Unpublished raw data.

Norenzayan, A., \& Hansen, I. (2006). Belief in supernatural agents in the face of death. Personality and Social Psychology Bulletin, 32, 174-187.

Peng, K., \& Nisbett, R. E. (1998). Cross-cultural similarities and differences in the understanding of physical causality. In M. Shield (Ed.), Proceedings of the Seventh Interdisciplinary Conference on Science and Culture (pp. 10-21). Frankfort, KY: Kentucky State University Press.

Pyysiäinen, I., \& Antonnen, V. (Eds.). (2002). Current approaches in the cognitive science of religion. New York: Continuum.

Rosch, E., Mervis, C., Grey, W., Johnson, D., \& Boyes-Braem, P. (1976). Basic objects in natural categories. Cognitive Psychology, 8, 382-439.

Rubin, D. C. (1995). Memory in oral traditions. Oxford, England: Oxford University Press.

Spelke, E. S. (1990). Principles of object perception. Cognitive Science, 14, 29-56.

Sperber, D. (1975). Rethinking symbolism. Cambridge, England: Cambridge University Press.

Sperber, D. (1996). Explaining culture: A naturalistic approach. Oxford, England: Blackwell.

Sperber, D., \& Wilson, D. (1986). Relevance: Communication and cognition (2nd Ed.). Cambridge, MA: Blackwell.

Stangor, C., \& McMillan, D. (1992). Memory for expectancy-congruent and expectancy incongruent information: A review of the social and social developmental literatures. Psychological Bulletin, 111, 42-61.

Thorndyke, P. W. (1977). Cognitive structures in comprehension and memory of narrative discourse. Cognitive Psychology, 9, 77-110.

Waddill, P. J., \& McDaniel, M. A. (1998). Distinctiveness effects in recall: Differential processing or privileged retrieval? Memory and Cognition, 26, 108-120.

Wellman, H. M., Cross, D., \& Watson, J. (2001). Meta-analysis of theory-of-mind development: The truth about false belief. Child Development, 72, 655-684. 